\title{
Drei Gesellschaften und ein Kongress
}

W er erinnert sich nicht gern an die überaus erfolgreiche erste gemeinsame Jahrestagung der allergologischen Gesellschaften ÄDA, DGAI und GPA im letzten September in Aachen? Die beiden Organisatoren Prof. Dr. Hans Merk und Dr. Frank Friedrichs haben uns spannende und interessante Tage bereitet mit einem großen Allergiekongress in Deutschland, wie es ihn in dieser Form mit Beteiligung der drei großen allergologischen Fachgesellschaften zuvor noch nicht gegeben hatte. Die fachlichen Bereicherungen und auch die zwischenmenschlichen Begegnungen werden sicherlich allen Teilnehmern in sehr angenehmer Erinnerung bleiben. Diese erfolgreiche Tagung hat wesentlich dazu beigetragen, dass sich Vertreter aller Gesellschaften Gedanken über zukünftige gemeinsame Kongresse gemacht haben. Wie heißt es so schön: Nur gemeinsam sind wir stark!

Anfang Mai fand dann eine Arbeitssitzung statt, in welcher der „Deutsche Allergie-Kongress" aus der Taufe gehoben wurde. $\mathrm{Ab}$ 2007 wird es jährlich nur noch eine große Allergietagung unter Beteiligung aller drei Fachgesellschaften in Deutschland geben! Die Federführung wechselt jährlich unter den drei Gesellschaften. Den Anfang - bzw. die Fortsetzung - wird Prof. Dr. Alexander Kapp von der DGAI in Hannover machen. Wir wünschen ihm und seinem Team viel Erfolg für die Vorbereitung.

Natürlich werden die drei Fachgesellschaften ihre Identität behalten und auch weiterhin die gerade für die Niedergelassenen so wichtigen regionalen Tagungen und Symposien veranstalten.

Gerade rechtzeitig vor Beginn der Schönwetterperioden mit intensiverer

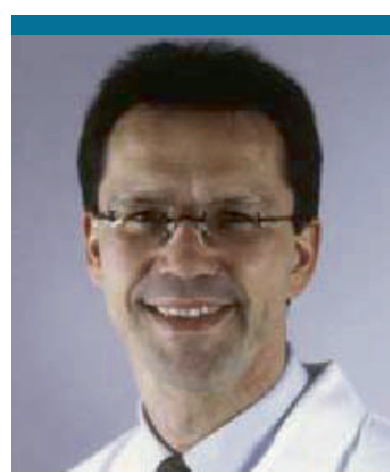

Prof. Dr. Ludger Klimek, Zentrum für Rhinologie und Allergologie, Wiesbaden, Vorstandsmitglied des ÄDA

schen photoprotektiven Effekten der bekanntesten Antioxidanzien Vitamin C und Vitamin $\mathrm{E}$ dargestellt.

In einer Kasuistik berichtet eine Arbeitsgruppe der Universitäts-Hautklinik Leipzig über den lehrreichen Fall einer anaphylaktischen Reaktion nach Kontakt mit Chlorhexidin-haltigen Gleitmitteln bei Einlage eines Blasenkatheters. Es bedarf aber sicherlich nicht einer urologischen Ausrichtung, um diesen Fall und die diesbezügliche Literaturübersicht mit großem Interesse zu lesen.

Sonneneinstrahlung wird in dem nun vorliegenden Heft des Allergo Journal in einem Beitrag über den möglichen protektiven Effekt der Vitamine C und E berichtet. Eine Arbeitsgruppe der Münchner Klinik und Poliklinik für Dermatologie und Allergologie am Biederstein gibt eine interessante Literaturübersicht zur Prävention akuter und chronischer Lichtschäden an der Haut durch Antioxidanzien. Hintergrund ist die Tatsache, dass der durch Ultraviolett-(UV-)Strahlung induzierte oxidative Stress zu Hautschädigungen führen kann. Basierend auf dem endogenen antioxidativen System könnte die Anwendung von Antioxidanzien, die reaktive Sauerstoffspezies inaktivieren, eine Erfolg versprechende Strategie in der Prävention sein. Diesbezüglich wird die Bedeutung von topischen und systemiChlorhexidinverbindungen sind weit verbreitete Antiseptika für die Hautund Schleimhautanwendung. Sie sind in fast allen gängigen Gurgellösungen im HNO- und Zahn-Mund-Kieferbereich enthalten wie auch in vielen Wundsprays, Gleitmitteln und sogar in Pudern und Kosmetika. Auch wir haben im HNO-ärztlichen Bereich in letzter Zeit mehrere vergleichbare Fälle gesehen, die die Bedeutung der vorliegenden Arbeit unterstreichen.

Des Weiteren möchten wir die Abstracts des 7. Europäischen Pollenflugsymposiums in Bad Lippspringe (11.-12. März 2005) Ihrer Aufmerksamkeit anempfehlen. Diese international ausgerichtete Tagung hat einige hochkarätige Informationen zur Pollenflugvorhersage zusammengetragen.

Wir wünschen Ihnen viel Spass bei der Lektüre,

Ihre

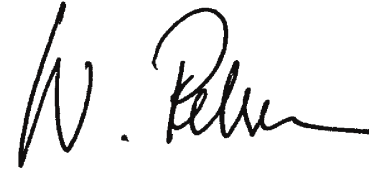

Dr. Wolfgang Rebien

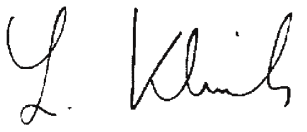

Prof. Dr. Ludger Klimek 